in vivo $33: 669-674(2019)$

doi:10.21873/invivo.11525

Review

\title{
Enhanced Recovery After Surgery: Is It Time to Change Our Strategy Regarding Laparoscopic Colectomy?
}

\author{
NIKOLAOS GARMPIS ${ }^{1 *}$, DIMITRIOS DIMITROULIS ${ }^{1 *}$, ANNA GARMPI ${ }^{2 *}$, \\ EVANGELOS DIAMANTIS ${ }^{3}$, ELEFTHERIOS SPARTALIS ${ }^{4}$, DIMITRIOS SCHIZAS ${ }^{5}$, \\ ANASTASIOS ANGELOU ${ }^{6}$, GEORGIOS-ANTONIOS MARGONIS ${ }^{7}$, \\ PARASKEVI FARMAKI ${ }^{8}$, EFSTATHIOS A. ANTONIOU $^{1}$, DIMITRIOS MANTAS ${ }^{1}$, \\ KONSTANTINOS MARKATOS ${ }^{9}$, KONSTANTINOS KONTZOGLOU ${ }^{1}$ and CHRISTOS DAMASKOS ${ }^{1}$ \\ ${ }^{1}$ Second Department of Propedeutic Surgery, Laiko General Hospital, Medical School, \\ National and Kapodistrian University of Athens, Athens, Greece; \\ ${ }^{2}$ Internal Medicine Department, Laiko General Hospital, Medical School, National and \\ Kapodistrian University of Athens, Athens, Greece; \\ ${ }^{3}$ Health Center Peristeriou, Athens, Greece; \\ ${ }^{4}$ N.S. Christeas Laboratory of Experimental Surgery and Surgical Research, Medical School, \\ National and Kapodistrian University of Athens, Athens, Greece; \\ ${ }^{5}$ First Department of Surgery, Laiko General Hospital, Medical School, National \\ and Kapodistrian University of Athens, Athens, Greece; \\ ${ }^{6}$ Alexandras Health Center, Athens, Greece; \\ ${ }^{7}$ Department of Surgery, The Johns Hopkins University School of Medicine, Baltimore, MD, U.S.A.; \\ ${ }^{8}$ First Department of Pediatrics, Agia Sofia Children's Hospital, National and \\ Kapodistrian University of Athens, Athens, Greece; \\ ${ }^{9}$ Biomedical Research Foundation (IIBEA), Academy of Athens, Athens, Greece
}

\begin{abstract}
Enhanced recovery after surgery or 'fast-track' methods are evidence-based protocols designed to standardize post-operative medical care, improve patient outcomes, promote early recovery, and reduce healthcare expenditure. Fast-track surgery is a multifunctional concept involving pre-, peri- and post-operative measures aiming to reduce the length of hospital stay and morbidity and complication rates, following elective abdominal surgery. Through the optimization of peri-operative care and the
\end{abstract}

This article is freely accessible online.

\footnotetext{
*These Authors contributed equally to this study.

Correspondence to: Dr. Nikolaos Garmpis, MD, M.Sc., Ph.D., Second Department of Propedeutic Surgery, Laiko General Hospital, Medical School, National and Kapodistrian University of Athens, 17 Agiou Thoma Street, 11527 Athens, Greece. Mobile: +306976291403, e-mail: nikosg22@hotmail.com
}

Key Words: Enhanced, recovery, ERAS, fast-track, laparoscopic, colon, surgery, review. recovery process in adherence to these fast-track protocols, improved outcomes are reached, surgical trauma and postoperative stress are reduced, with less surgical pain, reduced complications, and shorter length of hospital stay. Fast-track care requires a multidisciplinary collaboration of all healthcare professionals, as well as a high rate of protocol compliance and a good organizational structure. Despite the existing evidence of the benefits of fast-track protocols in a variety of surgical procedures and the similar outcomes of laparoscopic colonic surgery compared to open surgery, clear evidence of the benefits of fast-track care after laparoscopic colonic surgery is yet to be clearly demonstrated.

'Fast-track surgery' or 'enhanced recovery after surgery' (ERAS) was first introduced by Kehlet (1) in the 1990s and has been described abundantly thereafter. Fast-track surgery is a multimodal approach to patient care using a combination of several evidence-based peri-operative protocols to speed-up recovery after surgical interventions and integrates a plethora of modalities in surgery, anaesthesia, and nutrition. A multidisciplinary team is necessary to provide effective and safe fast-track methods (2). To date, fast-track surgery has yielded 
excellent results and there has been a significant reduction in hospital length of stay (LOS) without resulting in increased incidence of complications or re-admissions. The reported 2to 3-day reduction in LOS initially reported in available literature $(3,4)$ has been significantly improved over the years as the consensus guidelines for ERAS have been implemented (5). A number of randomized studies reported promising results favouring ERAS over conventional post-operative care $(6,7)$.

Any surgical procedures, including laparoscopic colonic surgery, affect a patient's normal physiology by causing a homeostatic imbalance, characterized by increased systemic metabolic response, also known as stress syndrome or stress response. This can lead to organ dysfunction, pulmonary and cardiac complications, muscle weakness and fatigue, fluid retention, electrolyte disbalance and ileus, leading to increased hospital LOS and substantial financial burden for the healthcare system.

In the recent past, patients who underwent colorectal surgery went through conventional peri-operative care, which consisted of sufficient mechanical bowel preparation, preoperative fasting, insertion of a nasogastric tube, insertion of multiple intra-abdominal surgical drains (SD), and postoperative fasting for up to 1 week. Since fast-track surgical protocols were introduced, surgeons have focused on easing post-operative pain and the peri-operative response stress, lowering the incidence of organ dysfunction, and encouraging earlier patient ambulation. All these parameters lead to positive outcomes; successful recovery after surgery, lower post-operative morbidity, shorter LOS, and less demand on healthcare resources. Since they were first introduced in the mid-1990s, fast-track protocols have gained ground as for as care of patients after colorectal surgery is concerned.

Fast-track protocols enforce early mobilization and feeding in order to reduce the procedure-related stress response of the patient, and exploits hospital resources to expedite the patient's recovery and discharge after undergoing colorectal surgery $(6,7)$. By employing a laparoscopic approach in combination with ERAS, one can accommodate a prompter return to function and faster discharge from hospital compared to those of traditional post-operative care. Post-operative morbidity rates are still high after elective colorectal resection and caution from surgeons tends to prolong LOS (8).

A significant number of surgeons nowadays consider laparoscopic colorectal surgery as the method of choice for a wide variety of colorectal pathologies. Level 1 evidence gathered from multicenter prospective randomized controlled trials led to adoption of laparoscopic surgery for colon cancer treatment, with acceptable results similar to that of open surgery in terms of feasibility, safety, and long-term outcomes $(1,9,10)$. However, these publications did not include any fast-track programs. Many evidence level 1 studies have highlighted that fast-track programs in conjunction with laparoscopic surgery are likely to promote recovery after surgery (11).

Colonic surgery is now considered to be totally distinct from rectal surgery, which requires deep pelvic dissection and is frequently followed by more complications (including distinct ones such as sexual dysfunction, urinary retention, pelvic organ injury, hypogastric nerve injury), complications that are not observed in abdominal colonic procedures, and a longer LOS. Moreover, compared with colonic segmental resection, rectal surgery has a higher degree of technical complexity, longer operative times, and use of retractors which is known to give rise to peri-operative morbidity. Consequently, patients undergoing rectal resection have not been included in previous studies involving fast-track programs (12).

The approach to fast-track surgery can be divided into three phases (13):

- Pre-operative phase: Patient selection and optimization.

- Peri-operative phase: Surgical and anaesthetic techniques to reduce procedure-related stress response of the patient.

- Post-operative phase: Multimodal approach to patient rehabilitation.

Pre-operative phase. Selection of patients is the first step to properly conducted fast-track modality and it is important to hold extensive discussion with candidate patients regarding the surgical process and the early rehabilitation programs. The selected patient would undergo evaluation of their performance status and, if necessary, would undergo an optimization process. Risk factor optimization is important as post-operative complications are usually linked to preexisting comorbidities $(12,14)$. Patients should actively participate in the evaluation and optimization processes. At this phase, patients should be informed of the benefits and disadvantages and be motivated to facilitate their recovery (15). All available information-providing methods, such as direct interviews, leaflets and multimedia, should be used.

One target of fast-track surgery is to improve surgical outcomes by eliminating the undesired surgical stress of the patient through reduction of post-operative peripheral insulin resistance. Therefore, ERAS emphasizes pre-operative management of carbohydrate intake. Proper patient assessment and counseling, and avoidance of premedication and carbohydrate overload prior to surgery take place during ERAS.

Human response to surgical or non-surgical trauma includes a variety of neuroendocrine changes that lead to retention of both salt and water, and catabolism of the existing stored fuels of the body. In 1930, Cuthbertson et al. first described the aforementioned surgical stress response and it later became clear that post-operative stress was milder compared to nonsurgical trauma response $(16,17)$. Kidney dysfunction and severe electrolyte disbalance of potassium, nitrogen, phosphorus, and sulphur ions were first reported in patients suffering from long bone fractures (18). Apart from the 
aforementioned changes, higher levels of adrenal cortical hormones as stress response were reported in experimental studies, although alternative nerve pathways limit this response (19). Surgical stress response consists of sympathetic nervous system stimulation, local cytokine secretion and production of additional catabolic hormones (20). The hypothalamicpituitary-adrenal axis is activated by increased cortisol and vasopressin secretion, in parallel with an elevated pancreatic secretion of glucagon. These changes lead to an upward trend in peripheral insulin resistance and further catabolism of skeletal muscle. Hyperglycemia, caused by peripheral insulin resistance, is believed to be an independent predictor of prolonged LOS and development of post-operative complications (21). All the response mechanisms are activated in an analogous manner to the initial triggering surgical trauma.

The aim of the pre-operative carbohydrate intake regime is to replicate normal metabolic response to eating breakfast, based on the concept that it stimulates endogenous insulin excretion, which down-regulates the metabolic state due to overnight fasting, and to some extent reduces the level of peripheral insulin resistance. Response to surgical trauma is accompanied by increased catabolism, muscle weakness, and more significant bacterial translocation compared to fed animals; thus, animals in the metabolically fasted state presented worse results than fed ones (22).

As a result of the aforementioned findings, pre-operative oral or intravenous administration of carbohydrates takes place up to 2 hours before surgery, in contrast to the traditional pre-operative fasting. Current evidence suggests the intake of $100 \mathrm{ml}$ of clear liquid beverage containing $12.5 \mathrm{~g}$ of polymers of carbohydrates that do not reduce osmotic load nor delay gastric dumping (23). The $100 \mathrm{ml}$ of this solution comprises $50 \mathrm{kcal}$, with osmotic pressure of $290 \mathrm{mOsm} / \mathrm{kg}$, while the $\mathrm{pH}$ is 5.0 . As reported by a recent study, a maximal volume of $400 \mathrm{ml}$ administered up to 2 hours before pre-operative opiate-containing analgesia led to a residual gastric volume equivalent to overnight fasting. So practically, the reason for keeping patients fasted does not exist. In this experimental study, insulin resistance was indeed reduced by either oral or intravenous peri-operative carbohydrate administration compared to overnight fasting in patients subjected to arthroplasty or colorectal surgery (24).

As becomes evident from the presented data, pre-operative carbohydrate administration reduces the post-operative development of insulin resistance and is commonly promoted as part of the processes of the ERAS pathway. These processes include routine neuraxial blockade, limited use of nasogastric tubes, limited or no use of SD, early post-operative ambulation and enteral feeding (25). Pre-operative administration of oral carbohydrate drinks, as opposed to the traditional pre-operative fasting increased patient comfort, reduced thirst and limited pre-operative anxiety as supported by a systemic review (22) and a recent randomized trial (26).
Despite the fact that it is established knowledge that preoperative carbohydrate administration reduces insulin resistance, there is still limited evidence supporting improvement in post-operative outcomes associated with pre-operative carbohydrate administration. A randomized trial concerning pre-operative intravenous administration of carbohydrates to patients planned to undergo cholecystectomy, reported no difference in clinical results, despite decreased insulin resistance (27). In a similar fashion, other studies on oral carbohydrate treatment in patients undergoing laparoscopic cholecystectomy reported no significant differences in clinical outcomes (28-30). Regarding colectomy, pre-operative and intra-operative intravenous glucose administration, as well as pre-operative oral carbohydrates benefited patients with an expedited recovery and reduced LOS (31-33). On the other hand, a large single-centre, randomized trial on elective liver resection and colorectal procedures demonstrated that oral carbohydrate administration did not lead to a significant improvement in post-operative fatigue or shorter LOS (30). As a conclusion, pre-operative carbohydrate administration is already established as part of fast-track surgery protocols, especially for colorectal surgeries, as existing data show a benefit for the patient. Further studies and randomized clinical trials are needed in order to extend this attitude towards more surgical procedures. However, some specific details of the pre-operative carbohydrate treatment have not yet been subjected to extensive analysis and thoroughly designed procedure-specific future studies are necessary.

Peri-operative phase. As fast-track programs are becoming more popular, especially for patients undergoing colon surgery, an international research group consisting of a number of institutions such as the European Society for Clinical Nutrition and Metabolism (ESPEN), the International Association for Surgical Metabolism and Nutrition (IASMEN), and the ERAS Society for Perioperative Care, have published guidelines for peri-operative care in elective colon surgery (34). During the procedure, the use of horizontal incisions or laparoscopic surgery minimize the patient's stress response. These outcomes are further improved in combination with one or more of the following peri-operative modalities: Rapidly acting anaesthetic agents, thoracic epidural anaesthesia, caution on salt and water overload, and active patient warming.

Currently, limited evidence exists regarding perioperative care in rectal surgery (35). Guidelines for perioperative care in elective rectal surgery were published by the ERAS Society for Perioperative Care, following the publication of colonic surgery guidelines. Based on them, it is considered that the application of ERAS principles refers to a particular number or subgroup of patients undergoing a rectal resection, as differences exist between colonic and 
rectal surgery outcomes (36). So far, the ERAS Society for Perioperative Care suggestions provide what is considered to be the evidence-based guidelines for each phase of the peri-operative treatment, despite the fact that these guidelines concern open rectal surgery and not laparoscopic procedures.

Post-operative phase. Traditionally, patients are transferred from the operating room to the intensive-care unit, then to high-dependency units and thereafter to the ward $(37,38)$.

By avoiding the use of nasogastric tubes and SD, and by promoting early oral fluid intake and early ambulation as part of the ERAS protocol, the patient's recovery highly depends on the post-operative analgesia. Especially in terms of ambulation and patient comfort, proper analgesic medication is critical (12). Post-operatively, ERAS patients are transferred to different post-operative care units which provide invasive monitoring. These units provide different levels of post-operative care depending on specific hospital settings and are given a variety of names: post-anesthesia care units, fast-track units, high-dependency units, step-down units, intermediate care units or intermediate care areas.

Post-operative ileus is a critical issue for the success of early rehabilitation, procedure-related morbidity, readmission rates and overall patient results. A number of different approaches to expediting post-operative bowel motility exist. Prokinetics (metoclopramide/domperidone), oral magnesium oxide, gum chewing, and bisacodyl suppositories have been evaluated and despite the lack of evidence that these agents are effective, a significant number of surgeons still use them as they claim to reduce time to bowel opening by a couple of days $(39,40)$.

The use of post-operative pelvic drainage after low anterior resection remains a controversial subject in modern surgery. Despite the fact that placement of SD is still preferred by a number of surgeons around the globe, it has not been proven by existing evidence to have any effect on the incidence of bloody ascites, anastomosis leakage of any other complication (34).

Fast-track discharge criteria are similar to those of traditional care pathways, but necessary steps to discharge a patient are performed earlier. Fast-track patient discharge is appropriate for some ambulatory afebrile patients with adequate food and liquid intake, gastrointestinal transit for gas, and effective pain control (41-43). No wound complications are allowed for fast-track discharge. Medical personnel should focus on preparing patients for their forthcoming discharge, to provide patient education on the surgeon's post-operative instructions and supply any prescribed medications for after discharge (5).

\section{Conclusion}

Fast-track recovery programs have a number of aims, including patient comfort, reduction in stress response to surgery, faster ambulation, shorter LOS, fewer re-admissions and speedy recovery. To accomplish all these aims, specific interventions are necessary in all phases of patient care. A number of publications report that laparoscopic colorectal surgery led to a re-admission rate of $10-15 \%$, similar to the respective rate in open colorectal resections (44-47). Nonsurgical complications also exhibited a significant decrease, while surgical complications did not follow this trend (48). Multiple randomized controlled trials and meta-analyses indicate that laparoscopic colectomy can lead to reductions in post-operative pain and ileus, preservation of normal pulmonary function, improved aesthetic result, shorter LOS by 2 to 3 days, and better quality of life when compared to open colorectal surgery (49-51). These short-term benefits of laparoscopic colectomy have been confirmed in recent Cochrane (52) and systemic reviews (53).

It is essential to implement detailed peri-operative care protocols based on level 1 evidence for early post-operative recovery of patients subjected to colorectal resection (11). In these studies, it was highlighted that patients who underwent these post-operative protocols demonstrated earlier bowel movement recovery, reduced LOS and fewer complications when compared to conventional postoperative care protocols. ERAS protocols improve the opportunity for rapid, uncomplicated post-operative recovery with significant benefits for patients while improving quality and saving money (54). It is obvious that ERAS benefits patients and healthcare systems alike, and further compliance with ERAS protocols will increase future benefits $(54,55)$.

Most evidence arising from previous studies concerned patients subjected to colonic surgery for a variety of pathologies, therefore standardization is required for early rehabilitation programs in rectal surgery. Only after these protocols have been validated using high-level evidence from randomized controlled trials can these protocols be applied in real-life clinical settings.

To date, post-operative care of patients who undergo laparoscopic surgery varies widely between countries, institutions and surgeons, and it is essential to revise present guidelines according to the specific needs of each subgroup of patients.

\section{Conflicts of Interest}

All the Authors declare that there is no conflict of interest.

\section{Authors' Contributions}

NG and CD designed the study. NG, DD, AG and CD wrote the article. ED, ES, DS, AA, GAM, PF and KM collected the data. NG, $\mathrm{DD}, \mathrm{AG}, \mathrm{EAA}, \mathrm{DM}, \mathrm{KK}$ and $\mathrm{CD}$ revised the article. 


\section{References}

1 Kehlet H and Wilmore DW: Fast- track surgery. Br J Surg 92: 3-4, 2005. PMID: 15635603. DOI: 10.1002/bjs.4841.

2 Zargar-Shoshtari K and Hill AG: Fast-track open colectomy is possible in a New Zealand public hospital. N Z Med J 121: 33 36, 2008. PMID: 18551151

3 Kehlet H: Fast-track colonic surgery: status and perspectives. Recent Results Cancer Res 165: 8-13, 2005. PMID: 15865015.

4 Singh PP and Hill AG: Fast-track elective colectomy: singlesurgeon experience of 100 consecutive cases. N Z Med J 126: 8-15, 2013. PMID: 23463105.

5 Slim K: Fast-track surgery: the next revolution in surgical care following laparoscopy. Colorectal Dis 13: 478-480, 2011. PMID: 21435146. DOI: 10.1111/j.1463-1318.2011.02589.x.

6 Hoffmann $\mathrm{H}$ and Kettelhack C: Fast-track surgery - conditions and challenges in postsurgical treatment: a review of elements of translational research in enhanced recovery after surgery. Eur Surg Res 49: 24-34, 2012. PMID: 22797672. DOI: 10.1159/000339859.

7 Lawrence JK, Keller DS, Samia H, Ermlich B, Brady KM, Nobel T, Stein SL and Delaney CP: Discharge within 24 to 72 hours of colorectal surgery is associated with low readmission rates when using enhanced recovery pathways. J Am Coll Surg 216: 390-394, 2013. PMID: 23352608. DOI: 10.1016/ j.jamcollsurg.2012.12.014

8 Trastulli S, Cirocchi R, Listorti C, Cavaliere D, Avenia N, Gullà N, Giustozzi G, Sciannameo F, Noya G and Boselli C: Laparoscopic $v s$. open resection for rectal cancer: a meta-analysis of randomized clinical trials. Colorectal Dis 14: e277-296, 2012. PMID: 22330061. DOI: 10.1111/j.1463-1318.2012.02985.x

9 Li P, Fang F, Cai JX, Tang D, Li QG and Wang DR: Fast-track rehabilitation $v s$. conventional care in laparoscopic colorectal resection for colorectal malignancy: A meta-analysis. World J Gastroenterol 19: 9119-9126, 2013. PMID: 24379639. DOI: 10.3748/wjg.v 19.i47.9119

10 Watt DG, McSorley ST, Horgan PG and McMillan DC: Enhanced recovery after surgery: Which components, if any, impact on the systemic inflammatory response following colorectal surgery?: A systematic review. Medicine 94: e1286, 2015. PMID: 26356689. DOI: 10.1097/MD.0000000000001286.

$11 \mathrm{Kim}$ DW, Kang SB, Lee SY, Oh HK and In MH: Early rehabilitation programs after laparoscopic colorectal surgery: Evidence and criticism. World J Gastroenterol 19: 8543-8551, 2013. PMID: 24379571. DOI: 10.3748/wjg.v19.i46.8543.

12 Rossi G, Vaccarezza H, Vaccaro CA, Mentz RE, Im V, Alvarez A and Quintana GO: Two-day hospital stay after laparoscopic colorectal surgery under an enhanced recovery after surgery (ERAS) pathway. World J Surg 37: 2483-2489, 2013. PMID: 23881088. DOI: $10.1007 / \mathrm{s} 00268-013-2155-\mathrm{x}$.

13 Nanavati AJ and Prabhakar S: Fast-track surgery: Toward comprehensive peri-operative care. Anesth Essays Res 8: 127133, 2014. PMID: 25886214. DOI: 10.4103/0259-1162.134474.

14 Khuri SF, Daley J, Henderson W, Hur K, Demakis J, Aust JB, Chong V, Fabri PJ, Gibbs JO, Grover F, Hammermeister K, Irvin G 3rd, McDonald G, Passaro E Jr., Phillips L, Scamman F, Spencer J and Stremple JF: The Department of Veterans Affairs' NSQIP: The first national, validated, outcome-based, risk-adjusted, and peercontrolled program for the measurement and enhancement of the quality of surgical care. National VA Surgical Quality Improvement Program. Ann Surg 228: 491-507, 1998. PMID: 9790339.
15 Chestovich PJ, Lin AY and Yoo J: Fast-track pathways in colorectal surgery. Surg Clin North Am 93: 21-32, 2013. PMID: 23177063. DOI: $10.1016 /$ j.suc.2012.09.003.

16 Cuthbertson DP: The disturbance of metabolism produced by bony and non-bony injury, with notes on certain abnormal conditions of bone. Biochem J 24: 1244-1263, 1930. PMID: 16744448.

17 Şimşek T, Şimşek HU and Cantürk NZ: Response to trauma and metabolic changes: posttraumatic metabolism. Ulus Cerrahi Derg 30: 153-159, 2014. PMID: 25931917. DOI: 10.5152/ UCD.2014.2653.

18 Wilmore DW: From Cuthbertson to fast-track surgery: 70 years of progress in reducing stress in surgical patients. Ann Surg 236: 643-648, 2002. PMID: 1422623. DOI: 10.1097/ 01.SLA.0000032942.79841.ED.

19 Little RA and Girolami A: Trauma metabolism - ebb and flow revisited. Br J Intensive Care 9: 142-146, 1999.

20 Desborough JP: The stress response to trauma and surgery. Br J Anaesth 85: 109-117, 2000. PMID: 10927999.Awad S and Lobo DN: Metabolic conditioning to attenuate the adverse effects of perioperative fasting and improve patient outcomes. Curr Opin Clin Nutr Metab Care 15: 194-200, 2012. PMID: 22157348. DOI: $10.1097 / \mathrm{MCO} .0 \mathrm{~b} 013 \mathrm{e} 32834 \mathrm{f0078.}$

21 Kratzing C: Pre-operative nutrition and carbohydrate loading. Proc Nutr Soc 70: 311-315, 2011. PMID: 21781358. DOI: 10.1017/S0029665111000450.

22 Wang ZG, Wang Q, Wang WJ and Qin HL: Randomized clinical trial to compare the effects of preoperative oral carbohydrate versus placebo on insulin resistance after colorectal surgery. Br J Surg 97: 317-327, 2010. PMID: 20101593. DOI: 10.1002/ bjs.6963.

23 Smith MD, McCall J, Plank L, Herbison GP, Soop M and Nygren J: Preoperative carbohydrate treatment for enhancing recovery after elective surgery. Cochrane Database Syst Rev 8: CD009161, 2014. PMID: 25121931. DOI: 10.1002/ 14651858.CD009161.pub2.

24 Burden S, Todd C, Hill J and Lal S: Pre-operative nutrition support in patients undergoing gastrointestinal surgery. Cochrane Database Syst Rev 11: CD008879, 2012. PMID: 23152265. DOI: $10.1002 / 14651858 . C D 008879 . p u b 2$.

25 Pimenta GP and de Aguilar-Nascimento JE: Prolonged preoperative fasting in elective surgical patients: Why should we reduce it? Nutr Clin Pract 29: 22-28, 2014. PMID: 24336400. DOI: $10.1177 / 0884533613514277$.

26 Nygren J, Thorell A and Ljungqvist O: Preoperative oral carbohydrate therapy. Curr Opin Anaesthesiol 28: 364-369, 2015. PMID: 25827282. DOI: 10.1097/ ACO.0000000000000192.

27 Bisgaard T, Kristiansen VB, Hjortso NC, Jacobsen LS, Rosenberg $\mathrm{J}$ and Kehlet $\mathrm{H}$ : Randomized clinical trial comparing an oral carbohydrate beverage with placebo before laparoscopic cholecystectomy. Br J Surg 91: 151-158, 2004. PMID: 14760661. DOI: $10.1002 /$ bjs. 4412 .

28 Hausel J, Nygren J, Thorell A, Lagerkranser M and Ljungqvist $\mathrm{O}$ : Randomized clinical trial of the effects of oral preoperative carbohydrates on postoperative nausea and vomiting after laparoscopic cholecystectomy. Br J Surg 92: 415-421, 2005. PMID: 15739210. DOI: 10.1002/bjs.4901.

29 Mathur S, Plank LD, McCall JL, Shapkov P, McIlroy K, Gillanders LK, Merrie AE, Torrie JJ, Pugh F, Koea JB, Bissett IP and Parry BR: Randomized controlled trial of preoperative oral carbohydrate treatment in major abdominal surgery. Br J Surg 97: 485-494, 2010. PMID: 20205227. DOI: 10.1002/ bjs.7026. 
30 Kanamori R, Shimamura M, Kinoshita M, Morioka N and Ozaki M: Preoperative carbohydrate administration prevents catabolism of fat and protein in patients undergoing elective laparoscopic colectomy: final report: 1AP5-10. Eur J Anaesthesiol 29(suppl 50): 17, 2012.

31 Fearon KC, Ljungqvist O, Von Meyenfeldt M, Revhaug A, Dejong CH, Lassen K, Nygren J, Hausel J, Soop M, Andersen J and Kehlet $\mathrm{H}$ : Enhanced recovery after surgery: a consensus review of clinical care for patients undergoing colonic resection. Clin Nutr 24: 466-477, 2005. PMID: 15896435. DOI: 10.1016/ j.clnu.2005.02.002.

32 Patel S, Lutz JM, Panchagnula U and Bansal S: Anesthesia and perioperative management of colorectal surgical patients - A clinical review (Part 1). J Anaesthesiol Clin Pharmacol 28: 162171, 2012. PMID: 22557737. DOI: 10.4103/0970-9185.94831.

33 Lei QC, Wang XY, Zheng HZ, Xia XF, Bi JC, Gao XJ and Li $\mathrm{N}$ : Laparoscopic versus open colorectal resection within fasttrack programs: An update meta-analysis based on randomized controlled trials. J Clin Med Res 7: 594-601, 2015. PMID: 26124904. DOI: $10.14740 /$ jocmr2177w.

34 Zhao JH, Sun JX, Gao P, Chen XW, Song YX, Huang XZ, Xu $\mathrm{HM}$ and Wang $\mathrm{ZN}$ : Fast-track surgery versus traditional perioperative care in laparoscopic colorectal cancer surgery: a meta-analysis. BMC Cancer 14: 607, 2014. PMID: 25148902. DOI: 10.1186/1471-2407-14-607.

35 Steenhagen E: Enhanced recovery after surgery: It's time to change practice! Nutr Clin Pract 31: 18-29, 2016. PMID: 26703956. DOI: $10.1177 / 0884533615622640$.

36 Zakhary WZA, Turton EW and Ender JK: Post-operative patient care and hospital implications of fast track. Eur Heart J Suppl 19(Suppl A): A18-A22, 2017. DOI: 10.1093/eurheartj/suw055.

37 Ender J, Borger MA, Scholz M, Funkat AK, Anwar N, Sommer M, Mohr FW and Fassl J: Cardiac surgery fast-track treatment in a postanesthetic care unit: six-month results of the Leipzig fast-track concept. Anesthesiology 109: 61-66, 2008. PMID: 18580173. DOI: 10.1097/ALN.0b013e31817881b3.

38 Nygren J, Thacker J, Carli F, Fearon KC, Norderval S, Lobo DN, Ljungqvist $\mathrm{O}$, Soop $\mathrm{M}$ and Ramirez J: Guidelines for perioperative care in elective rectal/pelvic surgery: Enhanced Recovery After Surgery $\left(\right.$ ERAS $\left.^{\circledR}\right)$ Society recommendations. Clin Nutr 31: 801816, 2012. PMID: 23062720. DOI: 10.1016/ j.clnu.2012.08.012.

39 Feldheiser A, Aziz O, Baldini G, Cox BP, Fearon KC, Feldman LS, Gan TJ, Kennedy RH, Ljungqvist O, Lobo DN, Miller T, Radtke FF, Ruiz Garces T, Schricker T, Scott MJ, Thacker JK, Ytreb $\varnothing$ LM and Carli F: Enhanced Recovery After Surgery (ERAS) for gastrointestinal surgery, part 2: consensus statement for anaesthesia practice. Acta Anaesthesiol Scand 60: 289-334, 2016. PMID: 26514824. DOI: 10.1111/aas.12651.

40 White PF and Song D: New criteria for fast-tracking after outpatient anesthesia: a comparison with the modified Aldrete's scoring system. Anesth Analg 88: 1069-1072, 1999. PMID: 10320170

41 Overdyk FJ, Harvey SC, Fishman RL and Shippey F: Successful strategies for improving operating room efficiency at academic institutions. Anesth Analg 86: 896-906, 1998. PMID: 9539621.

42 Duncan PG, Shandro J, Bachand R and Ainsworth L: A pilot study of recovery room bypass ("fast-track protocol") in a community hospital. Can J Anaesth 48: 630-636, 2001. PMID: 111495868.

43 Li LT, Mills WL, White DL, Li A, Gutierrez AM, Berger DH and Naik AD: Causes and prevalence of unplanned readmissions after colorectal surgery: a systematic review and meta-analysis.
J Am Geriatr Soc 61: 1175-1181, 2013. PMID: 23730901. DOI: 10.1111 /jgs.12307.

44 Goodney PP, Stukel TA, Lucas FL, Finlayson EV and Birkmeyer JD: Hospital volume, length of stay, and readmission rates in high-risk surgery. Ann Surg 238: 161-167, 2003. PMID: 12894006. DOI: 10.1097/01.SLA.0000081094.66659.c3.

45 Rochefort MM and Tomlinson JS: Unexpected readmissions after major cancer surgery: an evaluation of readmissions as a quality-of-care indicator. Surg Oncol Clin N Am 21: 397-405, 2012. PMID: 22583989. DOI: 10.1016/j.soc.2012.03.004.

46 Senagore AJ and Delaney CP: A critical analysis of laparoscopic colectomy at a single institution: lessons learned after 1000 cases. Am J Surg 191: 377-380, 2006. PMID: 16490550. DOI: 10.1016/j.amjsurg.2005.10.039.

47 Greco M, Capretti G, Beretta L, Gemma M, Pecorelli N and Braga M: Enhanced recovery program in colorectal surgery: a metaanalysis of randomized controlled trials. World J Surg 38: 15311541, 2014. PMID: 24368573. DOI: 10.1007/s00268-013-2416-8.

48 Chatterjee A, Chen L, Goldenberg EA, Bae HT and Finlayson SR: Opportunity cost in the evaluation of surgical innovations: A case study of laparoscopic versus open colectomy. Surg Endosc 24: 1075-1079, 2010. PMID: 19911224. DOI: 10.1007/ s00464-0090728-4.

49 Di B, Li Y, Wei K, Xiao X, Shi J, Zhang Y, Yang X, Gao P, Zhang K, Yuan Y, Zhang D, Wei X, Liu S, Wang J, Wang X, Zhang Y and Cai H: Laparoscopic versus open surgery for colon cancer: a meta-analysis of 5-year follow-up outcomes. Surg Oncol 22: e39-43, 2013. PMID: 23643698. DOI: 10.1016/ j.suronc.2013.03.002.

50 Gustafsson UO, Scott MJ, Schwenk W, Demartines N, Roulin D, Francis N, McNaught CE, MacFie J, Liberman AS, Soop M, Hill A, Kennedy RH, Lobo DN, Fearon K and Ljungqvist O; Enhanced Recovery After Surgery Society: Guidelines for perioperative care in elective colonic surgery: Enhanced Recovery After Surgery (ERAS ${ }^{\circledR}$ ) Society recommendations. Clin Nutr 31: 783-800, 2012. PMID: 23099039. DOI: 10.1016/j.clnu.2012.08.013.

51 Schwenk W, Haase O, Neudecker J and Muller JM: Short-term benefits for laparoscopic colorectal resection. Cochrane Database Syst Rev 3: CD003145, 2005. PMID: 16034888. DOI: 10.1002/14651858.CD003145.pub2.

52 Zhuang CL, Ye XZ, Zhang XD, Chen BC and Yu Z: Enhanced recovery after surgery programs versus traditional care for colorectal surgery: a meta-analysis of randomized controlled trials. Dis Colon Rectum 56: 667-678, 2013. PMID: 23575408. DOI: $10.1097 /$ DCR.0b013e3182812842.

53 Ljungqvist $\mathrm{O}$, Scott $\mathrm{M}$ and Fearon KC: Enhanced recovery after surgery: A review. JAMA Surg 152: 292-298, 2017. PMID: 28097305. DOI: 10.1001/jamasurg.2016.4952.

54 Kehlet H: ERAS implementation - Time to move forward. Ann Surg 267: 998-999, 2018. PMID: 29462010. DOI: $10.1097 /$ SLA.0000000000002720. 